\title{
Hermeneutik: matter of concern?
}

\author{
Martin Baisch
}

Eingegangen: 15. September 2021 / Angenommen: 20. September 2021 / Online publiziert: 7. Dezember 2021

(C) Der/die Autor(en) 2021

Zusammenfassung Der Beitrag umkreist zunächst aus der Perspektive der Lehre im Bereich der germanistischen Mediävistik Formen lesender Annäherung an das komplexe Erzählen im Bereich der mittelalterlichen Epik. Er plädiert vor dem Hintergrund der (zunehmenden) Diversität der Studierenden für ein Modell >langen Beschreibens $<$, das sich nicht vorschnell an überkommene Forschungspositionen kettet. Im Weiteren verfolgt der Essay anhand einer Skizze zur Forschungsgeschichte des Religiösen in Wolframs von Eschenbach Parzival die These, dass traditionelle hermeneutische Ansätze die unvermeidlichen habituellen Formungen und historischen Prägungen des forschenden Subjekts im Wissenschaftsprozess nicht erfassen können. Sie bedürfen der methodischen Ergänzung.

Schlüsselwörter Lesen · Lektüre · Beschreiben · Wolframs von Eschenbach Parzival und das Religiöse · Wilhelm Raabe

Martin Baisch $(\bowtie)$

Fachbereich Sprache, Literatur, Medien I, Institut für Germanistik, Fakultät für

Geisteswissenschaften, Universität Hamburg, Hamburg, Deutschland

E-Mail: martin.baisch@uni-hamburg.de 


\section{Hermeneutics: Matter of Concern?}

Abstract The article first circles around forms of reading approaches to complex narrative in the field of medieval epic poetry from the perspective of teaching in the field of German medieval studies. Against the background of the (increasing) diversity of students, it argues for a model of >long description< that is not rashly chained to outdated research positions. Furthermore, based on a sketch of the research history of the religious in Wolfram von Eschenbach's Parzival, the essay pursues the thesis that traditional hermeneutic approaches cannot capture the unavoidable habitual formations and historical imprints of the researching subject in the scholarly process. They require methodological supplementation.

Keywords Reading · Reading Process · Describing · Wolfram von Eschenbach's Parzival and the Religious · Wilhelm Raabe

\section{1 »Auch alle Fragen machen weiter, wie alle Antworten weitermachen«}

Der Winter naht: Auf den immerhin langen Sommer der Theorie - die wilden Jahre des Lesens - folgte der Herbst der Theorie, dessen Klarheit und Kühle alle Sehnsucht nach einer Rückkehr zu einstigen jugendlichen intellektuellen Abenteuern vertrieb und Melancholie und Erschöpfung diagnostizierte wie erzeugte. ${ }^{1}$ FAZ, Welt und NZZ lassen ihre angestellten Meisterschreiber in dürftigen Analysen in diesen Chor mit einstimmen. Wolfgang Thierse und Sarah Wagenknecht bitten empört um Ruhe: Es soll nicht mehr rappeln in der Kiste. Das kann man doch alles nicht mehr verstehen: gender-Gedöns und Multikulti. Das wird man doch nach sagen dürfen jeden Sonntag im Tagesspiegel Harald M. aus Kreuzberg. Der Winter naht. Nur auf die LiLi ist Verlass: Sie stemmt sich gegen diese restaurativ-apokalyptischen wie biedermännisch-normalisierenden Gesten der Absage an die Freiheit des Denkens, als wäre diese aufhebbar — und fragt nach einer neuen Hermeneutik und ihrer Notwendigkeit. Das hätte auch mutiger ausfallen können.

Fährt man mit dem taxi durch die gesellschaft oder hört und liest sich etwa durch Neil Young oder setzt sich Weil es sagbar ist aus oder trauert mit um Mein Jahr ohne Udo Jürgens, reibt man sich die Augen und wundert sich, wie man überhaupt darauf kommen kann vom Ende der Theorie zu raunen. ${ }^{2}$ Wer mit der Germanistik zu tun hat, weiß ohnehin, dass sie immer in der $\mathrm{Krise}^{3}$ ist - wie ja dies auch der eigentliche

\footnotetext{
1 Felsch, Philipp: Der lange Sommer der Theorie. Geschichte einer Revolte 1960-1990. München: C. H. Beck, 2015; Raulff, Ulrich: Wiedersehen mit den Siebzigern. Die wilden Jahre des Lesens. Stuttgart: Klett-Cotta, 2014; Poppenberg, Gerhard: Herbst der Theorie. Erinnerungen an die alte Gelehrtenrepublik Deutschland. Berlin: Matthes \& Seitz, 2018.

2 Nassehi, Armin: Mit dem Taxi durch die Gesellschaft. Soziologische Storys. Hamburg: Murmann, 2010; Erhart, Walter: Neil Young. Stuttgart: Reclam, 2015; Emcke, Carolin: Weil es sagbar ist. Über Zeugenschaft und Gerechtigkeit. Frankfurt a. M.: Fischer, 2015; Maier, Andreas: Mein Jahr ohne Udo Jürgens. Berlin: Suhrkamp, 2015.

3 Vgl. Schruhl, Friederike: Formationen der Praxis. Studien zu Darstellungsformen von Digital Humanities und Literaturwissenschaft. Göttingen: V\&R Unipress, 2020, S. 7-14.
} 
Zustand der Moderne war und ist. Als Mediävist*in ist Gelassenheit schon längst das Mittel der Wahl gegen die allgegenwärtige Abwertung etwa durch die Borniertheit der Suhrkamp-Kultur oder die kannibalistischen Praktiken von verbeamteten Beutegemeinschaften, die den nächsten großen Forschungsverbund eilfertig vorantreiben und das eine oder andere kleine Fach über die Klinge springen lassen, und muoze Antwort wie Untersuchungsgegenstand. Auch das Mittelalter macht weiter. Am Grunde der Moldau wandern die Steine./Es liegen begraben drei Kaiser in Prag.

\section{Wert der Hermeneutik}

»Gern wäre ich zum Beispiel auch Ordinarius für Tautologie an der Universität Kandersteg geworden. «4

Ob die Germanistik als Disziplin in ihrer Geschichte überhaupt etwas dazu beigetragen hat an produktiven Lösungen je aktueller Verstehens- und Verständnisprobleme in der Gesellschaft mitzuwirken, wäre eine Frage, die hier zu beantworten nicht der Raum ist. Viel eher aber, das lässt sich rasch feststellen, war sie beteiligt an oder stützte soziale, kulturelle und politische Legitimationsdiskurse lange Zeit unkritisch ( $>$ Nation $<$ ). Der damit angesprochene Aufsatz der Herausgeber in dieser Zeitschrift, den Autoren und Autorinnen zur Lektüre beigegeben, gibt sich besorgt um das in und seit der Aufklärung Erreichte, das gegenwärtig in der kulturellen Praxis scheitere und eine neue Hermeneutik erfordere. Die Digitalisierung, was genau damit gemeint ist, wird nicht expliziert, trage jedenfalls an der aktuellen Krise des Textverstehens die Schuld. Freilich wäre ohne Digitalisierung in den langen Monaten der Pandemie ein Gespräch über Texte und wie man sie verstehen könnte meist nicht möglich gewesen. Digitalisierung führe nur zu »simulierter Lektüre «, jedenfalls nicht zu echter, zu Lesen als »Konsum«, zu neuer »Unmündigkeit« an Schulen und Universitäten. Eine solche Klage hat topischen Charakter, sie wird schon lange geführt, wenn auch immer andere Ursachen für den Verfall angeführt worden sind. Lehrmaterialien, so behaupten die Herausgeber weiter, unterstellen, dass »Memorial- und Imaginationsmöglichkeiten« von Schülern und Schülerinnen vom »Smartphone beherrscht werden und so jedes mögliche Niveau von vorneherein unterlaufen«! Da ein Smartphone je nach Gebrauch schon recht >smart< sein kann, will man ergänzen und nicht dem Medium anlasten, das in seinen Möglichkeiten zu beobachten ist und in seinen Verwendungsweisen sorgsam zu regulieren wäre. Abhilfe schaffen soll auch hier eine >neue< Hermeneutik, die an Eco (Textintention) und Hermanns (Erkennen, Verstehen, Interpretieren) anschließen könne. Dass simulierte Lektüre als Regelfall universitärer Lehre anzusehen sei, weil »diese neuen Leser*innen « nicht anders könnten - dieser Befund will provozieren, denn er kann in seiner pauschalisierenden Wendung kaum richtig sein. ${ }^{5}$ Einer Form von Kulturkritik, die medienkritisch kon-

\footnotetext{
${ }^{4}$ Hildesheimer, Wolfgang: Mitteilungen an Max über den Stand der Dinge und anderes. Frankfurt a. M.: Suhrkamp, 1986, S. 23.

5 Näher am tatsächlichen Geschehen des Wandels des Lesens durch Formen der Digitalisierung scheinen mir die Beiträge in: Böck, Sebastian u. a. (Hg.): Lesen X.O. Rezeptionsprozesse in der digitalen Gegenwart.
} 
statiert, dass es kein richtiges Leben im falschen gibt, wünscht man alles und auch eine >neue Hermeneutik $<$, damit jene, die sie betreiben, sich wieder mit aller Kraft ihrer wichtigen Aufgabe widmen können: Jene auszubilden, die zu uns kommen, um ein Lesen zu lernen, das anderen Zwecken dient. Die Ressource, die im universitären Betrieb den Studierenden wie den Lehrenden und Forschenden mit allen Mitteln zu erhalten wäre, ist die der Zeit. Sie fehlt. Ihr Mangel scheint mir gegenwärtig das größte Problem an den Universitäten. ${ }^{6}$ Zuzustimmen, scheint mir, ist dem Befund, dass das Auftreten einer Vielzahl von Bindestrich-Hermeneutiken (etwa: New Virtual Hermeneutics), die für spezifische Forschungsgebiete entwickelt werden und auf besondere methodische Herausforderungen reagieren müssen, den Universalitätsanspruch der Hermeneutik als Verstehenslehre unterlaufen, die dieser in bestimmten Phasen ihrer Entwicklung zugesprochen worden ist. Für diese Entwicklung spielen eine Vielzahl von Faktoren eine Rolle, zu denen u. a. auch notwendige Ausdifferenzierungsprozesse des Wissenschaftssystems und das Streben nach Teilhabe an der hohen Geltung eines Begriffs bzw. einer Methodik gehören.

\section{Hermeneutik als matter of concern}

\section{»Hermeneutik ist, so verstanden, Instrument der Kritik. «7}

Einen Beitrag zur Theorie der Hermeneutik stellen meine Überlegungen nicht dar; sie versuchen vielmehr - einem Wunsch der Herausgeber folgend -, »den praktischen Stellenwert zu ermitteln, den das hermeneutische Methoden- und Wahrheitsverständnis« in meiner Lehre (und Forschung) zur älteren deutschen Sprache und Literatur spielt (wobei diese meist von Wahrheitsansprüchen entlastet sind). In seiner kleinen Schrift über das »Elend der Kritik« führt Bruno Latour die Unterschei-

Göttingen: V \& R unipress, 2017. Vgl. auch Michel, Sascha: Die Unruhe der Bücher. Vom Lesen und was es mit uns macht. Stuttgart: Reclam, 2020, S. 13-29. Den Körper des Lesenden analysiert in soziologischer Perspektive Perec, Georges: Denken/Ordnen. Zürich/Berlin: diaphanes, 2. Aufl. 2015, S. 103-120. Reflexionen über die fundamentalen Transformationen unserer Kultur durch das Digitale durchziehen das letzte Buch von Roberto Calasso etwa in Hinblick auf die Neugestaltungen des Wissens und ihre Ökonomien (Calasso, Roberto: Das unnennbare Heute. Berlin: Suhrkamp, 2019, S. 84-96).

${ }^{6}$ Das ist pathetisch formuliert. Und auch nur in Form der Klage. Aber sie berührt die gestellte Aufgabe: Lesen lernen im emphatischen Sinn und Methodiken des Verstehens zu reflektieren und einzuüben, benötigt eine gekonnte Didaktik, die auch dem Digitalen sich nicht mehr entziehen kann, aber ebenso Zeit, die nach der Bologna-Reform in ihrer Kostbarkeit neu wahrgenommen werden kann. Aber ist dieser Befund überhaupt richtig? Oder ist er nur angesteckt von dem immer wieder kehrenden Lamento der Überforderung durch die Zumutungen einer beschleunigten Gegenwart/Moderne? Wo es gesellschaftlich keine Mehrheit für ein Tempolimit auf Autobahnen gibt, wird man mit der Forderung nach >langsamen Lesen< kein Gehör finden. Zwischen Beschleunigung und Verlangsamung zu vermitteln, wäre vielleicht als Maxime hilfreich, um der Verknappung von Zeit entgegenzutreten.

7 So bekanntermaßen der letzte Satz der wegweisenden wie eleganten Studie von Szondi, Peter: Einführung in die literarische Hermeneutik. Studienausgabe der Vorlesungen Bd. 5. Frankfurt a. M.: Suhrkamp, 6. Aufl. 2012, S. 191. 
dung von matters of fact und matters of concern ein. ${ }^{8}$ Auf Heideggers Überlegungen zur Etymologie des Wortes >Ding< eingehend kommt Latour zu folgendem Schluss: »Einerseits ist ein Ding ein Objekt da draußen, andererseits ein Anliegen da drin$n e n$, in jedem Fall ein Versammeln. Um den von mir zuvor eingeführten Begriff jetzt genauer anzuwenden: dasselbe Wort Ding bezeichnet matters offact, Tatsachen, und matters of concern, Dinge, die uns angehen. ${ }^{9}$ Als ein $>$ Ding von Bedeutung $<$, abgekoppelt von jenen Versuchen, die bestimmen, was >Hermeneutik< als Tatsache ist, wird Hermeneutik hier praxeologisch beschrieben - in ihren Möglichkeiten, auch wo ihr nach meiner Auffassung methodisch Grenzen gesetzt sind.

Schon beim Erlernen der Übersetzungsfähigkeit aus dem Mittelhochdeutschen (oder aus dem Mittelniederdeutschen!) begegnet den Studierenden der mediävistischen Germanistik die Sprache, die sie täglich sprechen, als ein >fremdes-fernes< und gleichzeitig >eigenes-nahes< Phänomen, das sie ebenso fasziniert wie verzweifelt anzunehmen lernen. Sie erhalten zudem einen Einblick darin, ich beziehe mich hier auf Überlegungen von Paul Ricœur, dass beim Akt des Übersetzens das Verhältnis von Eigenem und Fremdem irreduzibel bestehen bleibt - »in der Erkenntnis des uneinholbaren Dialogizitätsstatus des Übersetzungsaktes als dem vernünftigen Horizont für das Verlangen nach Übersetzung. « ${ }^{10}$ Überdies erkennen sie, wie >Kritik< und >Hermeneutik<, die in den philologischen Entwürfen des 19. Jahrhunderts meist als methodisch getrennte Zugänge entworfen worden sind, beim Übersetzen untrennbar zusammengehören.

Im Folgenden gebe ich zunächst einen Bericht mit - wie mir scheint - symptomatischen Erfahrungen aus der Lehre, die das Angewiesensein auf hermeneutisch orientierte Verfahren zur Sicherung des Textverständnisses belegen und evaluieren. In einem zweiten Schritt versuche ich exemplarisch Hinweise zur Forschungsgeschichte des Parzival Wolframs von Eschenbach unter besonderer Berücksichtigung der Thematik des Religiösen zu geben. Diese zielen auf das Verhältnis von epistemischer und sozialer Seite von Wissenschaft und behaupten mehr als sie belegen, dass die Mittel hermeneutischer Verfahren endlich sind und begrenzt.

\section{Beschreiben}

Mein Seminar im Sommer 2021 über Suprematie und Anerkennung: Wolframs Kreuzzugsroman fand im dritten Corona-Semester nur das Interesse von vier Studentinnen. Wir einigten uns rasch darauf, die Gunst der Stunde zu nutzen, den von mir vorgelegten Seminarplan nicht zu beachten und dem Seminar eine neue Rahmung zu geben: In jeder Sitzung sollte Wolframs Romanfragment gelesen, Übersetzungsfragen geklärt und das ausgewählte Textstück hermeneutisch erschlos-

\footnotetext{
${ }^{8}$ Latour, Bruno: Elend der Kritik. Vom Krieg um Fakten zu Dingen von Belang. Zürich: diaphanes, 2021 [2007], S. 19. Latours Rede analysiert differenziert die gegenwärtige Krise des Text- und Weltverstehens in einem breiteren Horizont als die hier gestellte Frage nach einer Neohermeneutik.

9 Ebd., S. 23.

10 Ricœur, Paul: Vom Übersetzen. Herausforderung und Glück des Übersetzens. Berlin: Matthes \& Seitz, 2016, S. 17.
} 
sen werden. Lütflye Hammami, Madeleine Lauw, Kathrin Lüdeke und Franceska Vertullo erwiesen sich keineswegs als »neue Leserinnen«, von denen die Herausgeber der Zeitschrift ein so herzloses Bild entwerfen. Vielmehr zeigten sie sich als nachdenkliche und klug kombinierende Leserinnen, ausgestattet mit Neugier und Altgier, mit Übersetzungen, Kommentaren und Wörterbüchern, bereit sich in jeder Sitzung neu von Wolframs Erzählen im Willehalm in Staunen und Faszination versetzen zu lassen. Das Nicht-Verstehen dessen, was wir lasen, herrschte vor und erzeugte keineswegs Resignation, sondern im Gegenteil aus der Verwunderung über den fremdartigen Text den Wunsch mehr wissen und verstehen zu wollen. Wo wir bei Wolfram hinein stachen, erkannten wir im gemeinsamen Gespräch die komplexe Verwobenheit, den Austausch, die gegenteilige Behauptung innerhalb kleinster Versgruppen, Motiven und Metaphern im Text: So bei jenem Zweikampf, bei dem der junge christliche Märtyrer Vivianz vom heidnischen Gegner die Lanze mit dem Banner Amor in den Leib gestoßen bekommt und beide Krieger derart im Liebes- und Todeskampf verbunden sind. Doch schon vier Verse weiter ist von Minnedienst die Rede: Vom bartlosen Jüngling Vivianz, fragten wir uns erstaunt? Der aber, wie wir erfuhren, von der Fürstin Gyburc für die erste Schlacht ausstaffiert worden ist. Und auch jene Passage, in welcher der Erzähler Arabel/Gyburc anklagt, dass sie den Getauften wie den Nicht-Getauften großes Leid bringe und Schuld trage, irritierte uns; denn sofort erfolgte in der nächsten Verspartie eine revocatio: Wer den Mut habe, die Taufe zu nehmen, den Namen Christ anzunehmen und ebenso den neuen Namen Gyburg, sei ohne Schuld. Wie auch Maria, die immer Jungfrau war und bleibt und nur durch das Wort zu Jesus, ihrem Sohn, gekommen sei. Auf engstem Raum erzählt, vielfältig aufeinander bezogen und schillernd in den Bedeutungsnuancen: Zwei Lebensphasen kennen die beiden genannten außergewöhnlichen Frauen - markiert durch Namenswechsel und das >Wort<. Und so rau und verzweifelt erschien uns das Ende des ersten Buchs von Wolframs Roman, wenn der Fürst Willehalm durch die feindlichen Reihen der Heiden schleicht und in die Berge flieht, allein und verlassen, gescheitert, all die Toten hinter sich lässt aber doch noch das Pferd, das er im Kampf erworben hat, episch-grausam und rasch tötet, damit es nicht den heidnischen Feinden in die Hände fällt und ihnen nützen könnte. Dass Minneorientierung und -dienst sowie höfische Pracht der fremden Heiden als Zeichen adlig-höfischer Kultur im Erzählen so betont erscheinen, wiewohl die Folgen von Minnedienst, wie die anderen Werke Wolframs belegen, so destruktiv sind, hielten wir fest.

Ohne Blick auf die Einordnungs- und Klassifizierungsversuche der Forschung sahen wir inmitten des dichten Netzes von Motivkorrespondenzen, Figurenentwürfen, narrativen Sinnangeboten und Erzählmustermodellierungen, denen wir in der Lektüre begegneten und die wir gemeinsam zu beschreiben und erörtern versuchten, aus solchem Erzählen von vielfältigen Bedeutungsrelationen Momente des Unverbundenen herausragen - isolierte Figuren in ihren Einsamkeiten, Unverständlichkeiten und Brüchen, die nicht, so glaubten wir, als Leerstellen im eigentlichen, im literaturwissenschaftlichen Sinne zu füllen seien.

Wir waren mit den Mitteln der Hermeneutik als komplexem Lese- und Verstehensprozess Wolframs von Eschenbach Erzählen auf der Spur. Die Konstellation aus akademischem Wissen via langjähriger Forschungs- und Lehrtätigkeit zur hö- 
fischen Epik seitens des Seminarleiters (als eine Form von Habitualisierung von Wissen) und dem neuen Blick der Studentinnen der Germanistik mit je anderen sozialen und kulturellen Wahrnehmungsweisen und -hintergründen sowie intellektuellen Prägungen und Interessen ließ uns automatisch ein kritisches Verhältnis zu hermeneutischen Verstehensprozessen in unseren Sitzungen entwickeln - vor dem Hintergrund der so deutlich exponierten Alterität und Fremdheit des Wolframschen Erzählexperiments. ${ }^{11}$ Mit Bezug auf Nietzsche benennt Sheldon Pollock eine solche Form des Lesens als Gewahrsein der doppelten Bewegung, interpretieren zu müssen und Zurückhaltung zu üben gegenüber jeder Interpretation, um dem Gelesenen anerkennend und nicht aneignend zu begegnen. ${ }^{12}$ Damit ist ein verstehender Umgang mit literarischen Texten beschrieben worden, der sich grundsätzlich von dem unterscheidet, was Wolfgang Braungart über die Rezeption >schöner Stellen< und ihr Verhältnis zur literarischen Hermeneutik emphatisch entworfen hat. ${ }^{13}$ Sein Entwurf bekennt sich in jeglicher Hinsicht zu Konzeptionen von Literatur und Ästhetik seit dem 18. Jahrhundert, zu Subjektivität als Lesehaltung, das der bürgerlichen Epoche zugesprochen wird, doch aber nur dem >Leben< dienen soll. Belegen lässt sich dies exemplarisch durch das Plädoyer Braungarts für die >literarische Imagination $<$ ( $>$ Einbildungskraft $<$; $>$ Phantasie $<$ ), ohne die die $>$ Stelle $<$ nicht $>$ verlebendigt $<$ werden könne, worauf Literatur aber ziele. Schließlich: »In den Stellen finden wir uns wieder. $\ll^{14}$

11 Vgl. Reemtsma, Jan Philipp: Was heißt: einen literarischen Text interpretieren. Voraussetzungen und Implikationen des Redens über Literatur. München: C. H. Beck, 2016, S. 16 zur Legitimität von Formen habituellen Interpretierens literarischer Texte »in zureichend gebildeten Partialöffentlichkeiten«. Es erstaunt, dass der zugrundeliegende Literaturbegriff so konzeptualisiert erscheint, dass er die Literatur der Vormoderne und ihre Bedingungen konsequent ausblendet, obgleich die gewonnenen Erkenntnisse über den interpretativen Umgang mit Literatur ebenso konsequent generalisiert werden.

12 Pollock, Sheldon: Philologie und Freiheit. Berlin: Matthes \& Seitz, 2016, S. 24. Vgl. auch Felski, Rita: »Latour und Literary Studies«. In: Publications of the Modern Language Associations of America 130 (2015), S. 737-742, hier S. 740: »The task is to account for as many actors as possible, to be specific about forms of causation and connection (which are also forms of translation), instead of hitching a free ride on a preexisting theoretical vocabulary: the familiar isms waiting eagerly in the wings, all too ready to take on a starring role.«Susanne Köbele plädiert für den Umgang mit literarischen Texten, die von Phänomenen der Alterität geprägt sind, methodisch für das Mittel des geduldigen Beschreibens, sowie dem vorausgehend für eine schwebende Aufmerksamkeit. Vgl. Köbele, Susanne: »Zwischen Klang und Sinn. Das Gottfried-Idiom in Konrads von Würzburg Goldener Schmiede. (Mit einer Anmerkung zur paradoxen Dynamik von Alteritätsschüben.)« In: Anja Becker u.a. (Hg.): Alterität als Leitkonzept für historisches Interpretieren. Berlin: Akad.-Verl., 2012, S. 303-333.

13 Vgl. Braungart, Wolfgang: »Vom Sinn und Leben der Stelle.« In: Ders./Joachim Jacob (Hg.): Schöne, schöne Stellen. Oder: Wo das Verstehen beginnt. Göttingen: Wallstein, 2012, S. 64-143.

14 Ebd., S. 95. Stelle der Literatur und Lebenswelt des Lesenden konvergieren, so Braungart weiter, weil in der Stelle, die uns anspricht, »die Intentionalität des Kunstwerks und die Intentionalität des Lebens« (S. 106) aufeinanderträfen und sich verdichten würden. Was die Literatur feiern möchte, entpuppt sich als Akt der Verdinglichung und Auslöschung. Immerhin weiß der von Pathos geprägte Versuch, dass er für eine Philologie plädiert, in der die >Liebe < zum Wort eine Form angenommen hat, die von Narzissmus geprägt ist und von den Entwürfen zur ästhetischen Erfahrung bei Moritz und Kant abrückt, die »Selbstzurücknahme und Selbstbändigung « (S. 107) einfordern. Deren Ethik des Verstehens empfindet Braungart aber als »Anstrengung «, die er wohl nicht auf sich nehmen möchte (S. 107). Zu >erschöpft< erscheint hier die nach Resonanz und Resilienz strebende professorale Identität, die, um ihrer Selbstwirksamkeitserwartung zu entsprechen, im Akt des Lesens von >Stellen< den Status eines Parasiten nicht scheut. Dass ohne diese Anstrengung aber die >Begierde< ihren Gegenstand zerstört, ist jene Erkenntnis Schillers, die Wolfgang Riedel in seiner Abschiedsvorlesung betont und als Sublimierungsverlust analysiert. Vgl. Riedel, Wolf- 
Die Schreibgeste ist bewusst und unverkennbar nostalgisch - es wird zu verteidigen versucht, was als verloren empfunden wird.

Das andere Seminar, das ich im Sommer 2021 hielt, zur Thematik von Mittelalter und Film konnte nicht alle Studierende aufnehmen, die Interesse hatten. Zu jeder Sitzung waren die Kenntnis des Films und die Lektüre studentischer Essays erwünscht. Zu meiner Überraschung diskutierten wir in jeder Zoom-Sitzung 90 Minuten lang: Die Unterschiedlichkeit des Wahrgenommenen, die Interpretierbarkeit des Filmbildes, schließlich die varianten Deutungen des filmisch Erzählten auf der Ebene der Handlungsgestaltung und der Figurenkonzeption erzeugten Diskussionsbedarf. Kenntnisse aus dem Bereich der Filmgeschichte und der Film- und Medienwissenschaft, über die einzelne Studierende verfügten, bereicherten das Gespräch. In Terry Gilliams The Fisher King (1994) analysierten wir die Thematik von Empathie und Mitleid - in Rekurs auf Wolframs von Eschenbach Konzeption der compassio im Parzival. An der Interpretation von Udo Friedrich zu Bernard Taverniers La Passion de Beatrice (1987) kritisierten wir, dass hier ein Mediävist sein Mittelalterbild im Paradigma der Alterität auf einen Film projizierte, dem er Realismus und Authentizität attestierte - als ob dies je in einem Mittelalterfilm zu haben wäre. Dass Julie Delpy durch ihre Rolle als Beatrice im Weiblichkeitsmuster der Kindfrau zum Star des Kinos der 1980er Jahre wurde, ist der Art und Weise zu entnehmen, wie sie körperlich ins Bild gesetzt ist und weniger womit (Friedrich analysiert den Filminhalt: Beatrice mit Madonna). Die uns auffällige Gewaltdarstellung des Films ist keine Obsession, die an das Mittelalter zu binden wäre. Dass Delpy in ihrem nächsten Film - Schlöndorffs Homo Faber - wiederum in einem Fall von Inzest verwickelt wurde, muss im Kontext der Geschlechterpolitik des europäischen Kinos nach 1945 wahrgenommen werden. An Marketa Lazarova (1967) - der tschechischen >Nouvelle Vague< zuzurechnen - beeindruckte uns vor allem die Visualität der Bilder, die Zwang wie Schönheit des mittelalterlichen Christentums in komplexer Ikonographie ansichtig werden lässt. Der Winter blieb fern: An den Vergewaltigungsorgien von Game of Thrones zeigte niemand Interesse. ${ }^{15}$

\section{Gretchenfragen. Die Forschung zu Wolframs von Eschenbach Parzival auf der Suche nach sich selbst}

Die altgermanistische Forschung der späten 1940er und 1950er Jahre lenkte ihr Augenmerk bei ihrer Beschäftigung mit Wolframs Artusgralroman auf thematische Schwerpunkte wie >Schuld< und >Leid<. Das Erzählprogramm Wolframs - methodisch erschlossen durch Werkimmanenz und Hermeneutik - wurde auf die Formel gebracht, dass Ritter sein bedeutet, Fehler zu machen, Schuld auf sich laden, Sünden zu begehen, um diese in einem komplexen Erfahrungsprozess wieder gut zu machen.

gang: Ästhetische Distanz. Auch über Sublimierungsverluste in den Literaturwissenschaften. Würzburg: Königshausen \& Neumann, 2019.

$15 \mathrm{Vgl}$. ebenso in praxeologischer Perspektivierung und in Hinblick auf sich anschließende Schreibprozesse Martus, Steffen: »Interpretieren - Lesen - Schreiben.« In: Andreas Kablitz u.a. (Hg.): Hermeneutik unter Verdacht. Berlin/Bosten: de Gruyter, 2021, S. 45-82. 
Freilich konnte sich die germanistische Forschung dieser Jahre etwa bei Parzivals >Vergehen< nicht einig werden, wie diese zu verstehen seien und welches als seine $>$ schwerste Sünde< zu betrachten sei: Angeführt wurde der Hass gegen Gott (Friedrich Maurer), das Versagen auf der Gralsburg Munsalvaesche (Julius Schwietering), die Tötung des roten Ritters Ithers (Wolfgang Mohr) und der Tod der Mutter (Peter Wapnewski). Ebenso gelang es den damaligen Forschern nicht, die theologischen Referenzdiskurse zu identifizieren, auf die sich Wolfram von Eschenbach womöglich bezogen hat - von den literaturtheoretisch-methodologischen Schwierigkeiten, solche Bezugnahmen adäquat zu begründen und zu analysieren, ganz abgesehen.

Eine historisch begründbare und komplexe Motivationslage mag die Germanistik jener Zeit angetrieben haben, wenn ein Sachverhalt wie der Komplex einer >schuldlosen Schuld< so großes Interesse in der Forschung fand - lange Zeit vor dem Münchner Germanistentag von 1968, der es wagte, ein wenig Licht in die eigene Fachgeschichte zu werfen. In einem Portrait Helmut de Boors, der in den Jahren von 1930 bis 1945 in Bern Mittelaltergermanistik lehrte, ab 1949 an der Freien Universität Berlin einen Lehrstuhl innehatte, beschreibt Ulrich Wyss, Schweizer und einer anderen Forschergeneration angehörend, die Bewältigungs- und Erlösungsfunktion der Parzival-Figur für die damaligen Altgermanisten:

»In der Geschichte von dem Ritter, der als unwissender Tor in die Welt reitet, schuldlos schuldig wird, schließlich sich aus dieser Verstrickung herausarbeitet, hatte die Germanistik im Deutschland der fünfziger Jahre die Erfahrung ihrer eigenen Geschichte gespiegelt und inständig um das Verständnis von >Religiösität und Form < sich bemüht; die Bücher von Bodo Mergell, Gottfried Weber und Peter Wapnewski stehen dafür ein. $\ll^{16}$

Deutlich zeigten sich in diesen unabhängig voneinander entstehenden Versuchen die Grenzen einer Art von Hermeneutik, die »unter dem Mantel des Dialogischen Unverständliches und Diskontinuierliches zu marginalisieren [versuchte und] Interpretation als privilegiertes Verfahren ${ }^{17}{ }^{17}$ einsetzte, um je Deutungshoheit für das eigene Werk und über das Werk Wolframs zu gewinnen. ${ }^{18}$

\footnotetext{
16 Wyss, Ulrich: »Helmut de Boor (1891-1976)«. In: Christoph König/Hans-Harald Müller/Werner Röcke (Hg.): Wissenschaftsgeschichte der Germanistik in Porträts. Berlin: de Gruyter, 2012 [2000], S. 180-188, hier S. 186.

17 Kiening, Christian: Zwischen Körper und Schrift. Texte vor dem Zeitalter der Literatur. Frankfurt a. M.: Fischer, 2003, S. 27.

18 Nicht also erst Forschungsfelder wie die Disability Studies, die Queer und Postcolonial Studies sind »von einem außerwissenschaftlichen, nämlich gesellschaftspolitischen Interventionsziel her entworfen«. (Strohschneider, Peter: Zumutungen. Wissenschaft in Zeiten von Populismus, Moralisierung und Szientogratie. Hamburg: kursbuch.edition, 2020, S. 234) Daran scheint mir zunächst auch nichts verwerflich; andere Disziplinen der Geistes- und Sozialwissenschaften verfahren ebenso. Die Berücksichtigung der Kategorie gender führt (in meiner Lehre) zu einem komplexeren Verständnis der literarischen Werke und dient diesen. Und sie dient der Ausbildung der Reflexionsfähigkeit der Studierenden. Einem anderen Ziel folgen wir nicht. Wer behauptet, dass die Forschungsbemühungen dieser Fächer keinen methodischen Skeptizismus kennen würden und nicht geprägt seien von »intellektueller Irritabilität« (ebd., S. 234), erzeugt Unsicherheit im Verständnis der Argumentation. Es wird nämlich an dieser Stelle nicht präzise genug erläutert, wem dieser Vorwurf eigentlich gemacht wird: Judith Butler? Barbara Vinken? Andreas Kraß? James A. Schultz? Oder nur jenen, die den Protest gegen Sexismus und Rassismus an den amerikanischen Hochschulen organisieren? Fraglos verfügen diese Forschungsfelder über alle wissenschaftlichen Stan-
} 
Spätere Forschung verdrängte die Probleme um eine hermeneutische Erschließung und adäquate Interpretation der religiösen Sinnschichten des Parzival-Romans und ihres Verhältnisses zur höfisch-adligen Ordnung; sie reagierte auch nicht auf die Instrumentalisierung solcher Fragen durch die eigene Zunft. Der werkimmanent und hermeneutisch verfahrenden Germanistik wurde in den folgenden Jahren begegnet mit dem Versuch einer theoretisch-methodischen Öffnung. Das bedeutete für die Altgermanistik der 1970er Jahre (zumindest in der Lehre) einen Schub $>$ Entphilologisierung $<{ }^{19}$ aber auch die Hinwendung zu philosophisch und sozialwissenschaftlich orientierter Theoriebildung, die Bereitschaft zu Öffnung und Diskussion. Es brachte schließlich ebenso einen bunten Strauß an Legitimationsschwierigkeiten: Wozu noch mittelalterliche Literatur? Die sozialgeschichtliche Forschung dieser Zeit mit ihrem Interesse an den materialen Bedingungen des mittelalterlichen Literaturbetriebs (höfische Kultur, Gönnerforschung, Ministerialen-These) und den Formen und Formationen von Herrschaft und Macht konvergierte mit der Vorstellung, dass die im 12. Jahrhundert beginnende Literaturproduktion des höfischen Adels der Repräsentation von Herrschaft diene, auch Ausdruck sei von Bildungsstreben, Emanzipation und Säkularisierung, der Propagierung einer laikal geprägten Ethik. ${ }^{20}$ Die Hermeneutik, als Verfahren der Sinn- und Bedeutungserschließung eines literarischen Werkes wie auch durch ihre Vertreter theoretisch wie politisch unter Verdacht geraten, hatte einen schweren Stand.$^{21}$ Zumal das Paradigma der >Alterität<, angeregt durch die Einleitung einer Aufsatzsammlung eines der Gründungsprofessoren der Reformuniversität Konstanz, dem Romanisten Hans Robert Jauss, der mit 23 Jahren zum Hauptsturmführer der Waffen-SS aufstieg und im Oktober 1943 ei-

dards. Sie werden von Riedel (s. Anm. 14) in nämlicher Weise kritisiert: Geprägt von rigidem Moralismus würden sie zur »Mobilisierung phobischer Reflexe« (S. 44), im Studium der Geisteswissenschaften zu Bornierungseffekten und zu Bewertungszwang (S. 42) führen. Auffällig ist bei der Lektüre zunächst der hohe Affektgehalt, bei dem man sich fragt, weshalb hier die gepriesene Kulturleistung der Distanzgewinnung, die Erkenntnis benötigt, so dramatisch versagt.

19 Wyss, Ulrich: »Ich tuon sam der swan, der singet, swenne er stirbet. Über die Lesbarkeit des Minnegesangs«. In: Silvia Bovenschen (Hg.): Der fremdgewordene Text. Festschrift Helmut Brackert zum 65. Geburtstag. Berlin/New York: de Gruyter, 1997, S. 24-41, konstatiert S. 27 über den Traditionsbruch 1968 in Hinblick auf Textkritik und Editorik: »Man war es nunmehr leid, den Text immer wieder neu zu beschnuppern, ihn abzuschlecken, ihn am Ende zu zerkauen und sich dabei ungeheuer professionell zu dünken. Mit Lesen nämlich hatte derlei Geschäftigkeit nichts mehr zu tun. Das ästhetische Objekt, das sich in der Textkritik hätte konstituieren sollen, ging in ihr vollends verloren.«

20 Cramer, Thomas: »Normenkonflikte im Pfaffen Amis und im Willehalm von Wenden. Überlegungen zur Entwicklung des Bürgertums im Spätmittelalter«. In: ZfdPh 93 (1974), S. 124-140; Kaiser, Gert: »Der Wigalois des Wirnt von Gravenberc. Zur Bedeutung des Territorialisierungsprozesses für die >höfisch-ritterliche< Literatur des 13. Jahrhunderts«. In: Euphorion 69 (1975), S. 410-443; Peters, Ursula: »Artusroman und Fürstenhof: Darstellung und Kritik neuerer sozialgeschichtlicher Untersuchungen zu Hartmanns Erec«. In: Euphorion 69 (1975), S. 175-196; Kaiser, Gert: Textauslegung und gesellschaftliche Selbstdeutung. Aspekte einer sozialgeschichtlichen Interpretation von Hartmanns Artusepen. Wiesbaden: Akademische Verlagsanstalt Athenaion, 2. Aufl. 1978; Peters, Ursula: Literatur in der Stadt. Studien zu den sozialen Voraussetzungen und kulturellen Organisationsformen städtischer Literatur im 13. und 14. Jahrhundert. Tübingen: Niemeyer, 1983.

21 Erinnert sei nur an die kleine Schrift von Hörisch, Jochen: Die Wut des Verstehens. Zur Kritik der Hermeneutik. Frankfurt a. M.: Suhrkamp, 1988, die 2006, ergänzt durch Überlegungen zu Gadamers Wahrheit und Methode und zu der verfehlten Rezeption dekonstruktiv-antihermeneutischer Ansätze, neu aufgelegt worden ist. 
ne Kompanie befehligte, die in Kroatien Verbrechen gegen die Zivilbevölkerung durchführte, ${ }^{22}$ in den methodisch orientierten Debatten der Altgermanistik immer größere Bedeutung gewann. Als »Diskurs über die kulturell-historische (vertikale) Distanz zwischen Mittelalter und Moderne $\ll^{23}$ legitimierte sie ebenso die bedeutsame kulturwissenschaftliche Wende des Faches, die ihre hermeneutisch orientierte Forschungsmethodik nie völlig über Bord geworfen hat. In ihrer hegelianisch-geschichtsphilosophischen Ausrichtung überzeugte das Konzept weniger. Gründlich aufgearbeitet ist, wie sehr Jauss' Konzept der >Alterität $<$ selbst hermeneutischen Traditionen verpflichtet ist, wie auch innerhalb der altgermanistischen Theoriedebatte eine kritische Aufarbeitung von Begriff und Konzept geleistet worden ist, sowie Versuche der Weiterentwicklung dieses Ansatzes unternommen worden sind. ${ }^{24}$ Die Erforschung verschiedenster kulturwissenschaftlicher Thematiken, ebenso wie die Bemühungen um ein Verständnis mittelalterlicher Gesellschafts- und Kulturmuster, die hier unvollständig und ohne Nennung der Wegbereiter*innen mit den Stichworten Körper, Performanz und Präsenz, Medialität und Materialität (der Überlieferung), Genealogie und Verwandtschaft, Wissen, Emotion und gender benannt seien, sowie die Bereitschaft, das Fach interdisziplinär zu öffnen, ließen die germanistische Mediävistik an inneruniversitärem Prestige gewinnen.

Auch der >Rückkehr des Religiösen<, die sich seit den 2000er Jahren in unterschiedlichen Forschungszusammenhängen und -ausrichtungen auch in der Altgermanistik beobachten lässt, wohnt ihre Zeitbedingtheit und Zeitorientierung inne. Denn die Perspektive der Forschung, theoretisch oft versiert unterfüttert, richtete sich letztlich auf eine Begrifflichkeit von >Integration< und >Inklusion<des Fremden, seine Ein- und Unterordnung im dominanten christlichen Sinn- und Bedeutungszusammenhang, wie er exemplarisch vom Wolframschen Erzähler im Parzival wie im Willehalm virtuos und anspielungsreich entworfen zu sein scheint. Erst in jüngster Zeit mehren sich die Stimmen, die die germanistische Mediävistik bei ihren Forschungsbemühungen nicht nur bezogen sieht auf ihre eigenen historischen Ge-

\footnotetext{
22 Westermeier, Ursula: Hans Robert Jauss: Jugend, Krieg und Internierung. Konstanz: Konstanz Univ. Press, 2016.

23 Strohschneider, Peter: Art. »Alterität«. In: Klaus Weimar (Hg.): Reallexikon der deutschen Literaturwissenschaft. Neubearbeitung des Reallexikons der deutschen Literaturgeschichte. Bd. 1. Berlin/New York: de Gruyter, 1997, S. 58f., hier S. 58.

24 Hinweise bei Baisch, Martin: »Alterität und Selbstfremdheit. Zur Kritik eines zentralen Interpretationsparadigmas in der germanistischen Mediävistik «. In: Klaus Ridder/Steffen Patzold (Hg.): Die Aktualität der Vormoderne. Berlin: Akademie-Verlag, 2013, S. 185-206. Die Aufsätze von Christian Kiening zur Methodik altgermanistischer Forschung belegen seismographisch genau Wandlungen und Perspektivverschiebungen des Paradigmas der >Alterität< im Fach seit den 1990er Jahren: Kiening, Christian: »Anthropologische Zugänge zur mittelalterlichen Literatur: Konzepte, Ansätze, Perspektiven«. In: Forschungsberichte zur germanistischen Mediävistik 1 (1996), S. 11-129; ders.: »Alterität und Mimesis. Repräsentation des Fremden in Hans Stadens Historia«. In: Martin Huber/Gerhard Lauer (Hg.): Nach der Sozialgeschichte. Konzepte für eine Literaturwissenschaft zwischen historischer Anthropologie, Kulturgeschichte und Medientheorie. Tübingen: Niemeyer, 2000, S. 483-510; ders.: »Alterität und Methode. Begründungsmöglichkeiten fachlicher Identität«. In: Mitteilungen des Deutschen Germanistenverbandes 52/1 (2005), S. 150-166; ders.: »Literaturwissenschaftliche Mediävistik / Mediävistische Literaturwissenschaft«. In: Deutsche Vierteljahrsschrift für Literaturwissenschaft und Geistesgeschichte 89 (2015), S. 616-624.
} 
genstände, »sondern zugleich auf ihre eigenen postsäkularen Kontexte«. ${ }^{25}$ Die neue Aufmerksamkeit für die Leitkonzepte von >Geistlichem $<$ und > Weltlichem $<$ und ihrem Verhältnis zueinander, für Formen von Hybridisierungen und Ambiguisierungen, für die Fragen nach Herkünften, Wirkungsweisen und Funktionalisierung, sowie nach dem Emergieren von Pluralitätsbehauptungen belegt, dass das Methodenbewusstsein gegenüber früherer, positivistisch und geistesgeschichtlich orientierter Forschung zur Thematik gestiegen ist. Ob sie es schafft, endlich die Gretchenfrage für Wolframs >Parzival< hermeneutisch und methodisch angemessen $\mathrm{zu}$ beantworten, wird eine spätere Generation von Forschenden zu beantworten haben. Und das ist auch gut so.

\section{Distanzwahrungen und Näheforderungen}

\section{»Was hindert uns daran oder verbietet uns, >furchtbare Dinge zu wissen<? «"6}

Bei Schopenhauer findet sich der Gedanke, dass alle reflexive Achtsamkeit auf die Fallen der Vorannahmen des hermeneutischen Zirkels vergeblich sei, weil wir uns als Einzelne selbst fremd sind und bleiben. In der hier vorgelegten Skizze kann dies womöglich für den Fall Jauss konstatiert werden. Bei den anderen Beobachtungen sind eher politische Diskurseffekte, soziale und institutionelle Wandlungsprozesse (auch in der Institution der Universität) anzusetzen, die zu Veränderungen in den normativen und moralischen Einstellungen des >Homo Academicus $<$ führen und die womöglich unreflektiert bleiben, wenn etwa die Thematik des Religiösen in Wolframs Parzival Gegenstand der Forschung wird. Vor allem ist zu konstatieren, dass die Hinweise zur Forschungsgeschichte des Parzival keine moralisch-ethische Agenda besitzen, dass etwa mit echter oder gespielter Entrüstung auf die Verfehlungen früherer Forschergenerationen hingewiesen würde. Obgleich Ethik und die Freiheit von Forschung und Lehre, wie ich finde, nicht als strikte Gegensätze gedacht und entworfen werden müssen. ${ }^{27}$ Hermeneutik als textorientiertes, der Tradition zuge-

\footnotetext{
25 Hasebrink, Burkhard/Strohschneider, Peter: »Religiöse Schriftkultur und säkulare Textwissenschaft: Germanistische Mediävistik in postsäkularem Kontext«. In: Poetica 46 (2014), S. 277-291, hier S. $288 \mathrm{f}$. Vgl. auch Bernhardt, Susanne/Gebert, Bent (Hg.): Vielfalt des Religiösen. Mittelalterliche Literatur im postsäkularen Kontext. Berlin/Boston: de Gruyter, 2021.

26 Benjamin, Jessica: Anerkennung, Zeugenschaft und Moral. Soziale Traumata in psychoanalytischer Perspektive. Gießen: Psychosozial-Verlag, 2019, S. 14.

27 Eine solche Konzeption scheint bei Peter Strohschneider vorzuliegen (s. Anm. 18). Die Analysen zu gegenwärtigen Tendenzen sog. >Moralisierung < an den Universitäten in England und in den USA wären meiner Auffassung daraufhin zu befragen, worüber nicht gesprochen wird, was aber als die eigentlich prägenden Faktoren der Universität heute sind - auch und gerade in Hinblick auf die Frage nach Freiheit von Forschung und Lehre. Hier wären >Digitalisierung< und >Ökonomisierung< mit Sicherheit zu nennen. Wenn als Mittel der Wahl, um auf die genannten >Moralisierungen< verzichten zu können, von Peter Strohschneider u. a. >Takt< und >Anstand< empfohlen werden als Distanz bewahrende, die Fortsetzung von Kommunikation ermöglichende Kulturleistungen Alteuropas, möchte man ergänzen, dass diese Formen höfischen Verhaltenslehren entstammen, die bekanntermaßen moralische Ambivalenz nicht scheuen. Denn die in den Lehren behandelten Mittel der Verhaltensregulierung und -adaptierung sind strategisch einsetzbar: Es sind Mittel des Kampfes, eigene Interessen zu behaupten und Machtpositionen zu festigen jenseits bestehender Legitimitätssetzungen der sich entwickelten Institutionen. Wer je in einer universitären Berufungskommission mitgearbeitet hat, weiß um das destruktive Potential eben dieser Mittel, die in
} 
wandtes Verfahren der Verständnissicherung eines Textes scheint aber als methodisches Werkzeug stumpf, obgleich schon Szondi auf »die mannigfachen historischen Implikationen « hermeneutischer Verfahren hingewiesen hat. ${ }^{28}$

Es gibt aber andere methodische Instrumente, die etwa Soziologie und Politikwissenschaft bereitstellen und Abhilfe schaffen könnten. ${ }^{29}$ Mit Bezug auf Überlegungen des Indologen Shelden Pollock lässt sich diese Blindheit der literarischen Hermeneutik als eine ihr eigene Form des >Postkolonialismus< verstehen, der sich fatal gegen die eigene Tradition und deren Zeugnisse wendet. ${ }^{30}$ Weniger scheint mir die von Peter Strohschneider betonte Unterscheidung von institutioneller und epistemischer Seite von Wissenschaft eine Erklärung abgeben zu können. Vielmehr ist es die soziale Dimension von Wissenschaft und ihre Auswirkungen auf den Forschungsprozess, die durch die nicht in Frage zu stellenden Standards von Wissenschaft wie die Prinzipien der Distanz zum Untersuchungsgegenstand, der Vorbehaltlichkeit oder der Falsifikation nicht aus der (akademischen) Welt zu schaffen ist. Zu erinnern ist daran, dass während der Phase der Etablierung der Germanistik als Disziplin an den Universitäten des 19. Jahrhunderts das Berufsethos eines Professors und die methodische Ausrichtung der Germanistik als Philologie im Zeichen der Askese konvergierten. ${ }^{31}$ Das sog. >philologische Ethos< führte zur Heroisierung der eigenen Zunft und sicherte das instabile junge Fach ab, das sich an der Klassischen Philologie orientierte und eigene methodische Reflexion unterließ, die der Selbstvergewisserung des Faches und seiner Geltung hätte dienen können. In der Gattung der Philologenbiographien kulminierte das Bemühen, Forscher und Forschungsgegenstand und dessen philologische Erschließung als wesensgleich darzustellen und zu propagieren. Die soziale Dimension von Forschung wurde derart aufgewertet

den Klugheitslehren der Frühen Neuzeit beschrieben sind und Handlungsoptionen jenseits von Moralität reflektieren. Täuschung und Intrige, Verknappung von Information, der noch kürzere Dienstweg, der listige Austausch eines unliebsamen Kommissionsmitglieds, die Beauftragung eines bestellten Gutachtens, das mobbing eines widerständigen Kollegen sind amoralische Waffen der Klugheit, die trotz aller akademischen Vorkehrungen, Regulierungen und Prüfungen weiterhin wirksam sind und das Ziel, die Sicherung von Qualität in Forschung und Lehre, gefährden oder zerstören. Eine >Hermeneutik des Verdachts< kann hier nur die Prozesse der Delegitimierung jener Werte nachzeichnen, die sonst wohl von allen Mitgliedern der Kommission geteilt werden und meist zum Leitbild ihrer Institution gehören. Wenn der mit Stallgeruch berufen ist, hat sich der eigene Machtanspruch konsolidiert, ist metaphorisch unmissverständlich klar und bedarf keiner hermeneutischen Auslegung, dass die eingesetzten Mittel ihre Verwender stigmatisieren.

28 Szondi (s. Anm. 7), S. 25. Methodisch überzeugend leitet Szondi daraus die Notwendigkeit ab, hermeneutische Verfahren in einem auf Dauer gestellten Prozess zu reflektieren und zu evaluieren. Die Frage nach einer >neuen Hermeneutik< zielt auf den nämlichen Umstand.

29 Hier scheinen mir Überlegungen Walter Erharts (»Kommunikation, Anerkennung, Resonanz. Literaturwissenschaft und Kritische Theorie«. In: Andrea Albrecht u. a. [Hg.]: Literatur und Anerkennung. Wechselwirkungen und Perspektiven. Wien: LIT, 2017, S. 61-102) wegweisend, der in Axel Honneths Anerkennungstheorie ein Fundament sieht für eine zukünftige Gesellschaftstheorie, die eben deshalb für die Literaturwissenschaft anschlussfähig ist, weil ihr eine ästhetisch-literarische Theorie der Anerkennung zugrunde liegt. In ihrer Wahrnehmungsfähigkeit für soziale Pathologien kann sie die »Beschädigungen individueller Selbstverhältnisse« (S. 91) erfassen; damit wendet sie sich gegen Prozesse destruktiver Selbstfremdheit, die den oder die Einzelne/n wie auch das soziale Gefüge schädigen.

30 Pollock (s. Anm. 12), S. 47.

31 Vgl. zu den Ausführungen über die Germanistik als Disziplin im 19. Jahrhundert den wissenschaftssoziologisch orientierten Beitrag von Kolk, Rainer: »Wahrheit - Methode - Charakter. Zur wissenschaftlichen Ethik der Germanistik im 19. Jahrhundert«. In: IASL 14 (1989), S. 50-73. 
und gleichsam >unsichtbar $<$, als Gegenstand von (notwendiger) Selbstbeobachtung und Selbstreflexion jedenfalls nicht erkannt. Der hier nur angedeutete professorale Habitus, der im Fach der Germanistik sich entwickelte, hat sich in den statthabenden Modernisierungsschüben wie in den historischen Verwerfungen mit all ihren sozialen und politischen Folgen seither selbstverständlich transformiert, aufgelöst hat er sich nicht. $\mathrm{Zu}$ diesen gehört etwa der offensichtliche Geltungsverlust innerhalb der Gesellschaft; die als >Erniedrigung « wahrgenommene Einbindung in alle (!) Formen von Verwaltungsarbeit; ebenso, dass die Bindung zur eigenen Institution erkaltet ist (Klaus Heinrich). Dass diese Habitualisierung sich offenkundig bereitwillig durch außeruniversitäre Zugriffe und Vorgaben gestalten ließ oder lässt, wäre eine These, die ich nicht belegen kann. ${ }^{32}$ Mag man auch behaupten, dass Universitäten als Orte der Bildung von Selbstdistanz anzusehen seien, ${ }^{33}$ meint dies freilich nicht Selbstvergessenheit. Letztere entsteht womöglich leichter, wenn Selbstdistanz umschlägt in die Negation der Erfahrung von Verbundenheit: »Darum werden wir erst. « Hermeneutik als Methode hat hier die Aufmerksamkeitsleistung zu erbringen, ihr Verstehen zunächst zu begleiten, indem sie dieses Verstehen reflektiert, zudem erkennt sie, dass sie durch ihre Teilhabe am geschichtlichen Prozess mit dem zu erschließenden Gegenstand verbunden ist.

Wenn Joachim Heinzle in seiner jüngsten Monographie zu Wolfram von Eschenbach sich mit der zweiten Schlacht zwischen Heiden und Christen im Willehalm beschäftigt, ist $\mathrm{zu}$ beobachten, dass eine intensive und konkrete Auseinandersetzung mit der narrativen Brüchigkeit des Textes an dieser Stelle ausbleibt. Heinzles Ausführungen wenden sich rasch ab vom Erzählen der kriegerischen Geschehnisse; schnell beleuchten sie die Ebene des Politisch-Geschichtlichen, das der Willehalm auch anbietet (Karl und Roland; Pompeius - Weltherrschaft). Dabei wird doch soviel mehr erzählt! Was sich womöglich als Ausweichen vor dem so schwierig zu fassenden Erzählprozess im Willehalm beschreiben lässt, ist auch eine eminent hermeneutische Volte der Sicherung von Textverständnis: brüchigen Text zu einem Thema zu konzentrieren. ${ }^{34}$ Hier lässt sich nun unschwer anschließen an die Beobachtungen aus der Lehre: Was dort gelungen war, ist das emphatische Verbleiben auf dem Moment der Beschreibung des Erzählens im Willehalm und das Ausbleiben der hermeneutisch-interpretatorischen Zuordnung zu einer Begrifflichkeit bzw. Kategorienbildung, die invisibilisiert, was vielleicht der eigentliche Gegenstand des Textes ist, oder zumindest neue Beachtung verdiente. ${ }^{35}$

\footnotetext{
32 Roberto Calasso konstatiert, dass seiner Auffassung und Beobachtung nach die Kaste der Mitglieder der Universität durchgängig die Willensfreiheit des Menschen negieren und zugleich Wissenschaftsfreiheit einfordern und behaupten (Calasso [s. Anm. 5], S. 67).

33 Vgl. Strohschneider (s. Anm. 18), S. 242. Abstimmung und Aushandeln des Verhältnisses von Selbstbezug und Fremdbezug in einem permanenten Prozess (vgl. S. 243) scheinen mir eine andere Option anzudeuten.

34 Vgl. Heinzle, Joachim: Wolfram von Eschenbach: Dichter der ritterlichen Welt. Leben, Werke, Nachruhm. Basel: Schwabe, 2019, S. 207-213.

35 >Beschreibung< hier im Sinne der Differenzierung von >Analyse <, >Lektüre < und >Beschreibung < bei Kiening (s. Anm. 17), S. 28.
} 


\section{Entlastungen}

Unschwer lässt sich zwischen Paul Ricoeurs einflussreicher Studie zu Freud ${ }^{36}$ und seinen späten Werken zur Theorie der Anerkennung ein Band spannen: Seine berühmte Formel von der >Hermeneutik des Verdachts $<$ die er als methodischen Mechanismus in den Denkbewegungen von Freud, Marx und Nietzsche entdeckte und die die grundlegenden Wirkweise hermeneutischer Anstrengung von >hineinlegen< und >(hin)auslegen< verdeutlichte, hat in letzter Zeit Kritik erfahren. Denn es stellte sich die Beobachtung ein, dass neben angemessenen, aufdeckenden und aufklärerischen Interpretationsversuchen auch Formen >paranoischer $<$ Lektüren sich ausbildeten. In den Praxen vor allem der Literaturwissenschaften erkannte man, dass der $>$ Verdacht $<\mathrm{zu}>$ Verdächtigung $<$ und $>$ Enthüllung $<$ führte, $\mathrm{zu}>$ Deutungswahn $<$ (Aleida Assmann) und eben Paranoia - nämlich genau dann, wenn die >Hermeneutik des Verdachts< auf Dauer gestellt erscheint im Umgang mit literarischen Texten. Daher hat Eve Kosofsky Sedgewick vorgeschlagen, dass neben hermeneutischen Zugängen unter der Prämisse des Verdachts auch >reparative Lektüren< erprobt werden sollten. ${ }^{37}$ Das ließe sich auf die Formel einer >Hermeneutik der Anerkennung<bringen:

»Entgegen der Kritik, der Negation und des Zweifels, einer den literarischen Texten in den letzten Jahrzehnten häufig unterlegten >hermeneutics of suspicion<, die der Literatur gerade die Erkenntnis darüber entnimmt, was nicht funktioniert und nicht gelingt, gilt es heute vielleicht wieder andere, normativ und konstruktiv modellierte Funktionsweisen ästhetischer Erfahrung in den Blick zu rücken. Bereits Paul Ricœur hat gegen die Vorstellung eines Kampfes um Anerkennung die Idee von >Friedenszuständen< propagiert, in denen es zu einem temporären Ausgleich zwischen dem Selbstbezug und dem Recht des Anderen kommt. Ebenso könnte ein anderer literaturwissenschaftlicher Umgang mit der Vergangenheit literarischer Texte zu seinem Recht kommen, der zuletzt eher vernachlässigte soziale und kulturelle Praktiken der Lektüre und Interpretation berücksichtigt: die Bewahrung und die Sorge, die Vermittlung und Entfaltung ungeahnter semantischer Potentiale über Zeit und Raum hinweg, die Anschlussfähigkeit, Anhänglichkeit und Verbundenheit (>attachment $<$ ). «38

Angesichts der realen, zukünftig möglichen katastrophischen Ereignisse wie den drastischen Klimaveränderungen oder die Erosion der demokratischen Politikformen etwa durch Effekte der Digitalisierung, die die Erde und unsere Gesellschaften

\footnotetext{
${ }^{36}$ Ricoeur, Paul: Die Interpretation. Ein Versuch über Freud. Frankfurt a. M.: Suhrkamp, 1974.

37 Sedgewick, Eve Kosofsky: »Paranoid Reading and Reparative Reading, or, You're So Paranoid, You Probably Think This Essay Is About You«. In: Dies.: Touching Feeling: Affect, Pedagogy, Performativity. Durham, NC u. a.: Duke Univ. Press, 2003, S. 123-152.

${ }^{38}$ Erhart (s. Anm. 29), S. 101 f. Burkhard Hasebrink analysiert bei Meister Eckhart eine Konstellation, die strukturähnlich ist zu jener der >Friedenszuständeく bei Ricoeur: Indem der Gläubige sich notwendig und gänzlich von Gott entfernt, sich ihm entfremdet und ihn entbehrt, gibt er allen Selbstbezug auf und übergibt er die zukünftige Gestaltung der Verbindung dem Willen des Anderen. Vgl. Hasebrink, Burkhard: »Entbehrung des Anderen. Leiden als Paradigma der Alterität im Trostbuch Meister Eckharts«. In: Anja Becker u. a. (Hg.): Alterität als Leitkonzept für historisches Interpretieren. Berlin: Akademie-Verlag, 2012, S. 103-117.
} 
heimsuchen und nur wir zu verantworten haben, übersieht man leicht, dass jeder und jedem ein katastrophisches Ereignis im Leben sicher ist: der Umzug nach Altershausen. So jedenfalls entwirft es Wilhelm Raabes letztes Werk. Wie der Titel der Erzählung sagt, was ist, so auch der Name der Hauptfigur: Professor Dr. med. Geheimrath Friedrich Feyerabend. Titel- wie Namensgebung sind als ästhetische Signale zu verstehen, die darauf verweisen, dass in diesem literarischen Text eine $>$ Hermeneutik der Tiefe < bei ihren Bohrungen nichts finden wird. Auch ist etwa eine religiöse Absicherung oder Sinnstiftung nicht auszumachen: Reine Immanenz ist ein Prinzip dieses Erzählens! Berichtet wird, wie Feyerabend hoch geehrt seinen 70. Geburtstag feiert und dann grundlos aus allen Sinn- und Bedeutungszusammenhängen seines Lebens herausfällt. Er flüchtet in die Stadt seiner Herkunft und Kindheit und trifft in Altershausen auf seine greise Jugendfreundin. Der undramatisch erzählte Prozess der Erkenntnis, dass nach dem Erleben der Kindheit für alle Menschen ein Leben beginnt, das ein Leben im Falschen ist; und dass auch alle wissen, dass es ein Leben im Falschen ist. In der Wiederbegegnung mit der Stadt und der Freundin seiner Kindheit baut sich noch rascher alles ab. Geschichte und Tradition, der verdiente Ruhm seiner Karriere, das Hoffen durch die Rückkehr nach Alterhausen das verlorene Leben, dessen Sinn wiederzugewinnen: Alles scheitert. Gegenwart ist Vergangenheit, Vergangenheit ist Gegenwart: Ihre Differenz ist aufgehoben. Alle Grundlagen, Gegenstände, Funktionsweisen und Intentionen des Projekts der Hermeneutik sind in diesem am Ende des 19. Jahrhunderts entstandenen Fragment außer Kraft gesetzt. Die Mittel zum Verstehen sind es, das Verstehen selbst ist es auch. Es gibt nichts zu verstehen. Trost bietet, dass der Gestus des Erzählens geprägt ist von Empathie und Mitleid mit den erzählten Figuren. Und damit auch mit uns, den Lebenden und Lesenden. ${ }^{39}$

Funding Open Access funding enabled and organized by Projekt DEAL.

Open Access Dieser Artikel wird unter der Creative Commons Namensnennung 4.0 International Lizenz veröffentlicht, welche die Nutzung, Vervielfältigung, Bearbeitung, Verbreitung und Wiedergabe in jeglichem Medium und Format erlaubt, sofern Sie den/die ursprünglichen Autor(en) und die Quelle ordnungsgemäß nennen, einen Link zur Creative Commons Lizenz beifügen und angeben, ob Änderungen vorgenommen wurden.

Die in diesem Artikel enthaltenen Bilder und sonstiges Drittmaterial unterliegen ebenfalls der genannten Creative Commons Lizenz, sofern sich aus der Abbildungslegende nichts anderes ergibt. Sofern das betreffende Material nicht unter der genannten Creative Commons Lizenz steht und die betreffende Handlung nicht nach gesetzlichen Vorschriften erlaubt ist, ist für die oben aufgeführten Weiterverwendungen des Materials die Einwilligung des jeweiligen Rechteinhabers einzuholen.

Weitere Details zur Lizenz entnehmen Sie bitte der Lizenzinformation auf http://creativecommons.org/ licenses/by/4.0/deed.de.

39 Raabe, Wilhelm: Altershausen. Mit einem Nachwort von Andreas Maier. Berlin: Insel 2010. 